\title{
Blockchain and artificial intelligence for network security
}

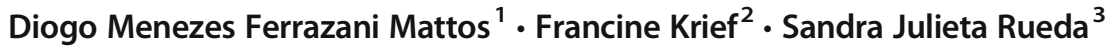

Published online: 29 January 2020

(C) Institut Mines-Télécom and Springer Nature Switzerland AG 2020

Blockchain is a disruptive technology that enables the development of reliable applications, without the need for trust between network peers. Blockchain technology creates global and immutable repositories that guarantee non-repudiation and accountability of stored information. Besides, the explosion in the generation and availability of data on computer networks raises the challenge of processing and managing large amounts of data with ever lower latencies. As a consequence, artificial intelligence and machine learning techniques experience significant improvements and emerge as enabling technologies for the next-generation networks. This special edition is dedicated to these new technologies that shape the world to have more reliable computer networks while enabling new distributed and knowledge-driven security applications and services. This issue's papers cover a wide range of topics, such as new cryptographic models applied to healthcare, intelligent threat-detection systems, and new consensus mechanisms for the blockchain.

After a thorough reviewing process, in which at least two experts have evaluated every paper, six papers have been accepted for this special issue. Reviewers' comments were helpful and productive first to select the most meaningful contributions as well as to improve the content, quality, and presentation of the accepted papers. Hereafter, we provide a summary of each paper in this special issue.

The first one from Marcela Tuler de Oliveira and her colleagues is entitled "A Break-Glass Protocol based on Ciphertext-Policy Attribute-Based Encryption to Access Medical Records in the Cloud." Although the electronic medical record (EMR) has to keep the patient's privacy, in the paper, the authors argue that the patient's EMR should be

Diogo Menezes Ferrazani Mattos

diogo_mattos@id.uff.br

\footnotetext{
Universidade Federal Fluminense, Rio de Janeiro, Brazil

Bordeaux INP, Bordeaux, France

Universidad de los Andes, Bogotá, Colombia
}

promptly available to healthcare professionals in the case of an emergency. Thus, the authors propose an attribute-based encryption protocol to provide access to the patient's encrypted EMR during acute stroke emergency treatment. The proposal assures authorization for accessing patient's data only for the emergency period. The authors prove that the proposed protocol is resilient to a comprehensive set of security threats, and they also expose the usability of the protocol in a real-world scenario.

The second paper from Hélio do Nascimento Cunha Neto and his colleagues is proposing the "MineCap: Super Incremental Learning for Detecting and Blocking Cryptocurrency Mining on Software-Defined Networking." The authors claim that unallowed cryptocurrency mining is a current threat in corporative networks. Hence, the authors model the discovery of cryptocurrency mining as a classification problem on a machine learning approach. Moreover, Cunha Neto et al. propose a new incremental learning scheme, called super incremental learning, in which offline candidate classifiers feed a super classification model. The supermodel performs incremental learning on the samples' pertinence probability for each candidate model and each target class. The authors show growth in the supermodel accuracy as the proposal learns with new arriving data.

The following paper from Fabio Cesar Schuartz and his colleagues is entitled "Improving Threat Detection in Networks Using Deep Learning." In this paper, the authors address the threat detection in big streaming data traffics. The authors propose a deep learning method as a data preprocessing layer to reduce the data dimensionality before the data analysis in the stream processing layer. The proposal applies a deep learning neural network, which automatically extracts the feature from an original feature dataset. The proposal reduces the complexity of the original feature set and, thus, implies higher accuracy, lower false-positive and falsenegative rates, and faster classification time.

The fourth paper from Vanessa Chicarino and her colleagues focuses "On the Detection of Selfish Mining and Stalker Attacks in Blockchain Networks." The authors ponder 
that bifurcations, or forks, naturally arise in the blockchain until it reaches a consensus. Nevertheless, malicious users may force the occurrence of forks to profit from network rewards or to hamper the revenue of a specific user. The authors identify two primary malicious behaviours, the selfish miner and stalker, and propose heuristics on the blockchain height to detect attacks. Simulation results show that proposed heuristics are accurate in recognizing that the blockchain network is under attack.

The next paper from Samuel Masseport and his colleagues is entitled "Proof of Usage: User-centric consensus for data provision and exchange." The authors introduce a new consensus protocol for private and permissioned blockchain networks. The proposed protocol encourages users to spend coins in the network to have a chance to mine the next block of the chain. Proof-of-usage protocol is an alternative to the proofof-work protocol and shares principles with both proof-ofstake and proof-of-activity. One of the key outcomes of the proposed consensus protocol is that, while rewarding users that actively use the blockchain, it keeps the currency stable over time. The proposal is practical, and it is currently deployed in a real blockchain platform.

The last paper from Gabriel Reis Carrara and his colleagues is entitled "Consistency, Availability and Partition Tolerance in Blockchain: A Survey on the Consensus Mechanism over
Peer-to-Peer Networking." The authors note that blockchain, as any distributed system, is subjected to the CAP theorem (Consistency, Availability, and Partition Tolerance) and, thus, any consensus mechanism on blockchain assures at most two out of the three properties despite one of them. Therefore, the authors survey the consensus proposals on blockchain networks and identify which properties each consensus proposal satisfies. Moreover, the paper enlightens the differences between probabilistic and deterministic consensus mechanisms. The authors also reason for drawbacks and the costs of each consensus mechanism to the blockchain network.

Acknowledgements The guest editors would like to express their deep appreciation to the editor-in-chief, Prof. Guy Pujolle, for giving them the opportunity to publish this special issue. The guest editors also thank the managing editor, Alexia Kappelmann as well as the journal editorial staff for their continuous support during the process of this publication. Last, the guest editors would like to express their thanks to all the authors for submitting quality articles and the reviewers for helping in the selection of papers and improving the accepted papers.

Publisher's note Springer Nature remains neutral with regard to jurisdictional claims in published maps and institutional affiliations. 